\begin{tabular}{|c|c|}
\hline Title: & $\begin{array}{l}\text { Measurement of inverter caused losses in permanent magnet synchronous machines } \\
\text { using a modular multiphase multilevel converter }\end{array}$ \\
\hline Authors: & $\begin{array}{l}\text { Christoph Rollbühler, Lukas Stefanski, Stefan Gretzinger, Johannes Kolb, Marc Hiller, } \\
\text { Martin Doppelbauer }\end{array}$ \\
\hline Institute: & $\begin{array}{l}\text { Karlsruhe Institute of Technology (KIT) } \\
\text { Institute of Electrical Engineering (ETI) } \\
\text { Hybrid Electric Vehicles (HEV) }\end{array}$ \\
\hline Type: & Conference Proceedings \\
\hline Published at: & $\begin{array}{l}\text { Proceedings } 2019 \text { 10th International Conference on Power Electronics and ECCE Asia } \\
\text { (ICPE } 2019 \text { - ECCE Asia) } \\
\text { Publisher: IEEE } \\
\text { Year: } 2019 \\
\text { ISBN: } 978-89-5708-313-0 \\
\text { Pages: } 1239-1245\end{array}$ \\
\hline Hyperlinks: & https://ieeexplore.ieee.org/document/8796933 \\
\hline
\end{tabular}

(C) 2019 IEEE. Personal use of this material is permitted. Permission from IEEE must be obtained for all other uses, in any current or future media, including reprinting/republishing this material for advertising or promotional purposes, creating new collective works, for resale or redistribution to servers or lists, or reuse of any copyrighted component of this work in other works. 


\title{
Measurement of inverter caused losses in permanent magnet synchronous machines using a modular multiphase multilevel converter
}

\author{
C. Rollbühler ${ }^{1 *}$, L. Stefanski ${ }^{1}$, S. Gretzinger ${ }^{1}$, J. Kolb $^{2}$, M. Hiller ${ }^{1}$ and M. Doppelbauer ${ }^{1}$ \\ ${ }^{1}$ Institute of Electrical Engineering, Karlsruhe Institute of Technology, Karlsruhe, Germany \\ ${ }^{2}$ SHARE at KIT, Schaeffler Technologies AG \& Co. KG, Karlsruhe, Germany \\ * E-Mail: c.rollbuehler@kit.edu
}

\begin{abstract}
Current distortion of two-level converter-fed permanent magnet synchronous machines causes additional machine-side losses, which lead to a distinct reduction in the drive train efficiency of electric vehicles for instance. Determining these losses by measurement is still an ambitious task. On the one hand, there's a lack of adequate voltage sources. On the other hand, there is no accurate procedure to distinguish between the different loss components. In this publication, a $100 \mathrm{kVA}$ seven-level multilevel converter is used to determine the losses of a $60 \mathrm{~kW}$ highly-utilized permanent magnet synchronous machine for automotive use. Due to the good voltage quality of the multilevel converter, the machine currents have very low harmonic distortion and the determined losses are considered as fundamental ones. In a second experiment, the machine is operated with a conventional two-level converter. Comparing machine losses in both experiments allows the determination of the additional machine losses in two-level operation.
\end{abstract}

Index Terms - Loss Measurement, Multilevel Converter, Interior Permanent Magnet Synchronous Machine, Current Ripple

\section{INTRODUCTION}

In medium- and high-voltage applications, multilevel converters (MLC) are a very popular converter family. They are often used as motor- or grid-side converters and offer many advantages due to their modular and scalable structure. Furthermore, MLCs provide a good output voltage quality and accordingly are well suited to determine the fundamental parameters of electrical machines. In comparison to linear amplifiers, which are also used widely for investigations on fundamental parameters, MLCs allow higher power ratings due to the switched operation of the semiconductors. In comparison to synchronous generators MLCs have no small frequent harmonics in voltage due to slotting or similar effects.

The application for machines is shown in previous work [1-4], where the impact on the machine's behavior was rarely investigated. In [5], the total harmonic distortion (THD) of induction machine currents and the temperature of the machine windings for sinusoidal voltage supply and an eleven-level Modular Multilevel Converter (MMC) are observed and discussed. In [6], induction motors are examined in no-load and full-load tests supplied by an eleven-level MMC and a generator. Due to harmonics caused by slotting effects of the generator, the generator causes more losses in the motor than the multilevel converter. Further investigations on iron losses under multilevel-voltage excitation are made in [7], where ring core samples are measured with two-, three- and five-level voltage inverters. The measurements with the ring core samples are then used for finite-elementbased investigations: The effects of different voltage waveforms are shown and the losses are separated and compared. In [8], inverter caused induction machine losses, fed with two- and three-level voltage inverters, are compared. However, only no-load operation is examined.

Summing up, the given references show that machine losses decrease as the number of voltage levels increases. Yet a method of measuring these additional losses over the whole operating range of the machine is not shown so far. Most publications focus on induction machines and are limited to particular operating points or no-load operation, e.g. [9]. Furthermore, these approaches lack separating the additional losses in magnetic and copper losses by measurement.

This paper presents a new method of measuring the additional machine losses caused by the voltage harmonics of the inverter in two-level operation. The idea is to compare the same operating point of the machine with different feeding voltage waveforms. Here, a two-level and a seven-level inverter are used to operate a highly utilized interior permanent magnet synchronous machine (IPMSM). By comparing the measured losses, the additional inverter-caused machine losses in two-level operation are determined and separated in additional magnetic and additional copper losses. The separated loss shares are used to improve the machines loss model. The method is also applicable for other three-phase machines.

\section{THEORY}

\section{A. Fundamental Machine Model}

The machine has three symmetrical star connected phases with the neutral point not connected to the inverter. The stator voltage equations are identified by applying Ohm's law, Faraday's law of induction, and Kirchhoff's laws to the machine coils. After transforming to the rotorfixed reference frame the dq-voltage equations of the IPMSM result: 


$$
\begin{aligned}
& v_{\mathrm{d}}=R \cdot i_{\mathrm{d}}+L_{\mathrm{dd}} \frac{\mathrm{d} i_{\mathrm{d}}}{\mathrm{d} t}+L_{\mathrm{dq}} \frac{\mathrm{d} i_{\mathrm{q}}}{\mathrm{d} t}-\omega \psi_{\mathrm{q}} \\
& v_{\mathrm{q}}=R \cdot i_{\mathrm{q}}+L_{\mathrm{qq}} \frac{\mathrm{d} i_{\mathrm{q}}}{\mathrm{d} t}+L_{\mathrm{qd}} \frac{\mathrm{d} i_{\mathrm{d}}}{\mathrm{d} t}+\omega \psi_{\mathrm{d}}
\end{aligned}
$$

$R$ describes the phase resistance, $L_{\mathrm{xy}}$ the machinespecific inductances, $\omega$ the electric angular frequency, $t$ the time, and $v_{x}, i_{x}$ and $\psi_{x}$ the voltages, currents, and flux linkages in the direct and quadrature axes with $x, y \in$ $\{d, q\}$. Harmonic effects due to slotting, manufacturing asymmetries as well as inverter nonlinearities have not been taken into account.

\section{B. Voltage Generation}

As this paper focuses on the influences of the two-level inverter switching, the generation of the required voltages $v_{\mathrm{d}}$ and $v_{\mathrm{q}}$ must be considered. Assuming ideal sinusoidal voltages, $v_{\mathrm{d}}$ and $v_{\mathrm{q}}$ are constant in stationary operation. Due to the switching behavior of the inverter, $v_{\mathrm{d}}$ and $v_{\mathrm{q}}$ are not constant during one switching cycle and a current ripple is present. The extent of the current ripple depends on aspects such as the DC-voltage, modulation method and switching frequency of the converter and the operatingpoint dependent values of the inductances. In Fig. 1, the inverter output voltage waveforms of one phase of twoand seven-level operation are shown exemplary. Since the seven-level output voltage shows a better accordance to sinusoidal voltage, the resulting current ripple is lower at the same operating point of the machine. Generally, the number of voltage levels is obtained from the phase voltage of a given converter structure; the output voltage levels in this paper are counted similarly to maintain comparability to other publications in this field of expertise. Thinking of inverter caused current ripple though, the causal voltage is line-to-line voltage, which has $2 n+1$ levels, thus three levels in two-level operation and thirteen levels in seven-level operation $(n$ is the number of converter branches, $n=1$ in two-level and $n=6$ in seven-level operation.).

\section{Machine Losses}

The main loss quantities in electrical machines are copper, magnetic and mechanic losses. In copper, the
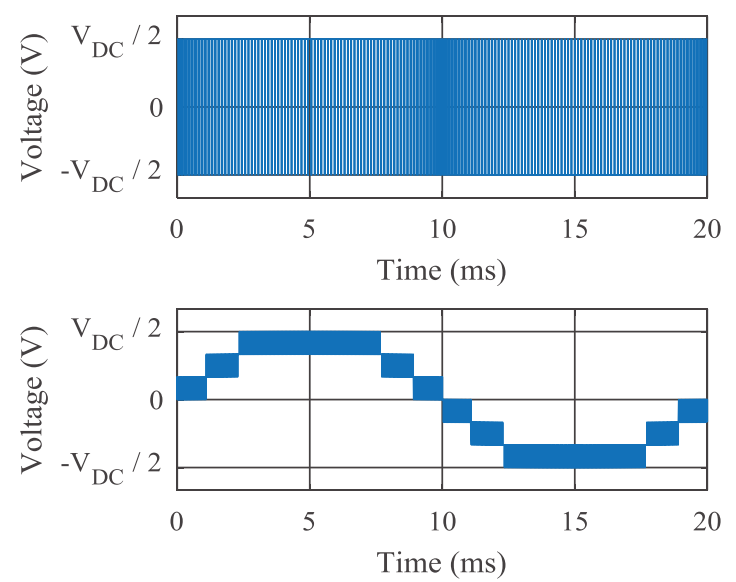

Fig. 1. Output voltage waveforms for two-level (upper half) and seven-level (lower half) operation with $V_{\mathrm{DC}}=300 \mathrm{~V}$. current causes thermal losses, defined by

$$
P_{\mathrm{Cu}}=\frac{1}{T_{\text {per }}} \int_{0}^{T_{\text {per }}} R(\vartheta) \cdot i^{2}(t) \mathrm{d} t
$$

A current ripple increases the RMS-value of the machine's current which results in higher ohmic losses $P_{\mathrm{Cu}}$. Additional losses due to skin and proximity effect are not considered as the machine has a distributed winding and a relatively low maximum electric frequency of $500 \mathrm{~Hz}$.

Losses in the magnetic domain of IPMSMs are divided into iron and magnet losses. Iron losses are caused by eddy currents and domain wall movements in the steel sheets. Many models exist for modeling iron losses in different specimen [10-13]. However, prediction in complex geometries is still an ambitious task. Iron losses depend on the actual magnetic field, the actual amount of flux in the specimen and its frequency. Additional harmonics in current cause so-called minor loops in the magnetic hysteresis curve, which result in additional iron losses [10] and harmonics in the flux itself. Furthermore, eddy currents occur in the machine's permanent magnets, since high frequent components in flux cause induced voltages in the rotor and therefore in the rotor's magnets. As the magnets are not separated in axial direction, eddy currents result and lead to significant magnet losses in the rotor. Separating iron and magnet loss shares is merely possible through FE-analysis or calorimetric measurements, since the permanent magnets in the rotor are not accessible during machine operation.

\section{Simulation RESUlts}

In advance of the measurements, simulations of the given machine (see TABLE I) fed by a two- and a sevenlevel voltage source were performed to gain knowledge of the expectable current ripple in the machine. The specific behavior of the current ripple depending on the operating point is studied and the estimated reduction due to sevenlevel operation is determined. Furthermore, additional copper losses can be evaluated in simulation.

TABLE I MACHINE DATA

\begin{tabular}{l|c|c|}
\multirow{2}{*}{} & \multicolumn{2}{|c|}{ IPMSM } \\
Nom. & Max. \\
\hline Current in A & 169 & 300 \\
\hline Torque in Nm & 130 & 220 \\
\hline Speed in rpm & 4000 & 10000 \\
\hline Pole pairs & \multicolumn{2}{|c|}{3} \\
\hline$R_{\mathrm{S}}\left(20^{\circ} \mathrm{C}\right)$ in $\Omega$ & \multicolumn{2}{|c|}{10.5} \\
\hline Weight in $\mathrm{kg}$ & \multicolumn{2}{|c|}{51.5} \\
\hline DC-voltage in $\mathrm{V}$ & \multicolumn{2}{|c|}{300} \\
\hline
\end{tabular}

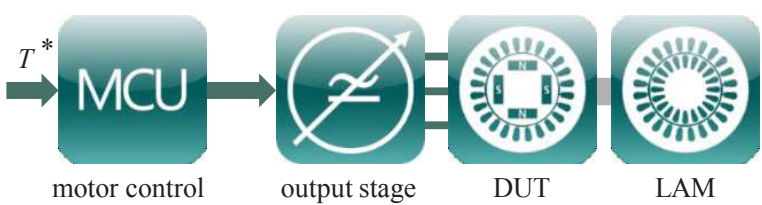

Fig. 2. Structure of the simulation model 


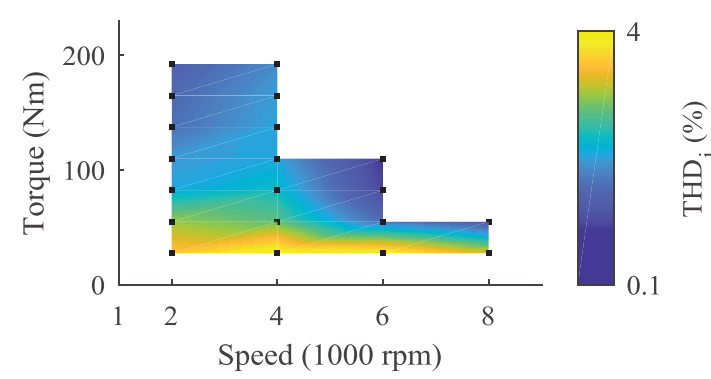

(a) Two-level operation

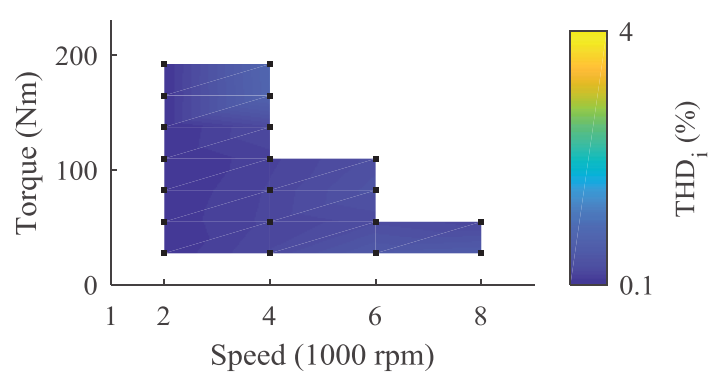

(b) Seven-level operation

Fig. 3. Machine currents THD, markers represent simulated operating points.

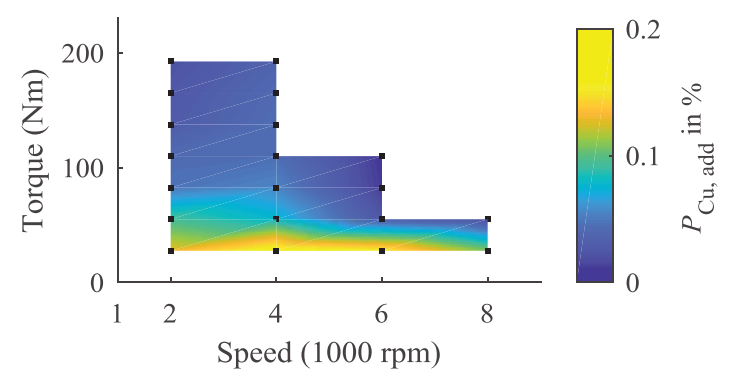

(a) Two-level operation

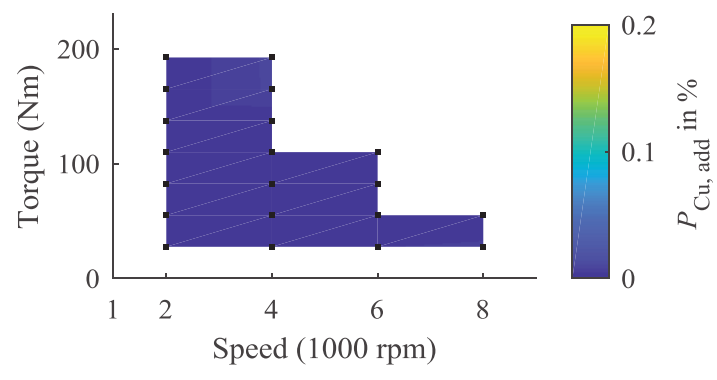

(b) Seven-level operation

Fig. 4. Additional copper losses compared to fundamental copper losses of two-level and seven-level operation. Markers represent simulated operating points.

The simulation model in Fig. 2 shows a motor control unit (MCU) which generates reference values and controls a non-causal modeled IPMSM in Matlab Simulink Simscape [14]. The IPMSM, called device under test (DUT), is fed by a variable voltage output stage. An induction machine controlled at constant speed serves as the load machine (LAM). The variable voltage output stage generates a multilevel voltage with an adjustable number of voltage levels. In this investigation, two- and seven-level operation are compared and exemplary output voltage waveforms are shown in Fig. 1. The MCU is set to operate at a control period of $125 \mu \mathrm{s}$. However, the modulation of the output stage depends on the number of output voltage levels. In two-level operation, it is operated at $8 \mathrm{kHz}$. In seven-level operation, each of the six submodule converters operates at $20 \mathrm{kHz}$ on average, resulting in an overall output switching frequency of $120 \mathrm{kHz}$. Since inverter losses are not investigated, the different switching frequencies are not relevant for the comparison. In fact, the higher switching frequency of the MLC is a positive side-effect for reducing harmonic content.

The simulations are performed with the same DCvoltage, control-period, switching frequencies and machine parameters as those of the test bench, which is introduced in IV. The parameters in TABLE I are fundamental machine parameters, flux characteristics are obtained from measurements and stored in lookup tables [14]. The voltage equations of the machine are given in (1) and (2). Since the simulation only considers copper losses and fundamental iron losses, no predictions of additional inverter-caused iron losses can be made by simulation.
The simulation shows the estimated reduction of the current ripple in multilevel operation. In Fig. 3, the THD of the phase currents of the electric machine was determined for several operating points in two- and sevenlevel operation. In seven-level operation, current ripple is reduced by up to 40 times, so the remaining harmonic content in the phase currents can be neglected. This indicates the suitability of a seven-level MLC for the measurement of fundamental losses in an IPMSM. Generally, THD is low at higher torque demands, as the greater fundamental component in current dominates the increasing current ripple due to saturation of the inductances at higher current values. Furthermore, the current ripple is lower at lower speeds $(<1000 \mathrm{rpm})$. Due to the short active state of the inverter, the time for a current change is short compared to higher speeds. In field weakening mode (>4000 rpm) the THD is decreasing, because the currents fundamental itself is increased due to a demanded negative d-current.

The harmonic copper losses in two-level and sevenlevel operation are minimal due to the low harmonic content of the currents. Fig. 4(a) shows the percentage of the harmonic copper losses $P_{\mathrm{Cu} \text {,add }}$ for two-level excitation, which are even lower in seven-level operation, Fig. 4(b). Since less than $0,2 \%$ of the total copper loses are harmonic copper losses, they are not significant. However, the current distortion cannot be ignored in the case of magnetic losses. Current ripple causes high frequent magnetic minor loops, which cause significant additional losses in iron. For instance, [7] predicts an iron loss reduction in ring cores of up to $40 \%$ changing from two- to a five-level feeding voltage. The determination of 
additional iron losses is performed in the experimental chapter.

Summing up, the simulations show that seven-level operation reduces the current ripple to a negligible value. The measured machine losses in the experimental chapter can therefore be assumed to be fundamental losses and the additional losses caused by the two-level inverter can be derived.

\section{EXPERIMENTAL SETUP}

An overview of the test bench setup is given in Fig. 5 . A $60 \mathrm{~kW}$ IPMSM of type HSM1-6.17.12-Col from Brusa serves as the device under test, the machine properties are given in TABLE I. A speed-controlled induction machine by Wittur is used as load machine. Self-developed twolevel converters for power supply are based on Semikron six-pack modules SKiiP 513GD122-3DUL, see Fig. 5. Since the DUT requires a reduced DC-voltage of $300 \mathrm{~V}$, one converter serves as DC/DC converter and supplies motor converter 1 . Motor converter 1 is connected to the DUT and can either be a two-level converter or a sevenlevel MLC. Measurements are performed using a 12 bit LeCroy HD 4101 oscilloscope, PMK BumbleBee differential probes and Tektronix A6304XL current transducers at $20 \mathrm{MSps}$ to ensure proper accuracy in measuring the multilevel converters currents and voltages. To make sure that only machine losses are measured, voltages are tapped directly at the machine's terminals.

Since motor converter 1 is used as measurement converter of the DUT in two- and seven-level operation, it has two output stages: a conventional two-level stage based on the Semikron module mentioned above and a seven-level modular multiphase multilevel converter (MMPMC), which was introduced in [15]. The converter topology is shown in Fig. 6. The MMPMC consists of six branches per phase. Each branch itself consists of an IGBT half-bridge per phase and a flux compensated choke at the output. By switching the top or bottom IGBT of every branch, the multilevel output voltage is generated by means of an inductive voltage divider with a very low output inductance, see [15]. Therefore, load-dependent voltage drops are neglectable. Since the multilevel output voltage modulation is performed with $120 \mathrm{kHz}$, every single branch of the inverter is switched with an average of $20 \mathrm{kHz}$. Thus, the MMPMC is an excellent voltage source, capable of operating the IPMSM under nearly sinusoidal conditions, as shown in section $\mathrm{V}$.

The converter system (including both output stages) is controlled by a digital signal processing system based on the digital signal processor (DSP) TMS320C6748 produced by Texas Instruments, [16]. Lookup tables of the fundamental flux linkages of the machine are stored in an external SD-RAM. The lookup tables are used to parameterize the predictive current control of the machine, which was introduced in [17]. The control frequency is equal to $8 \mathrm{kHz}$ for both, two- and seven-level operation.
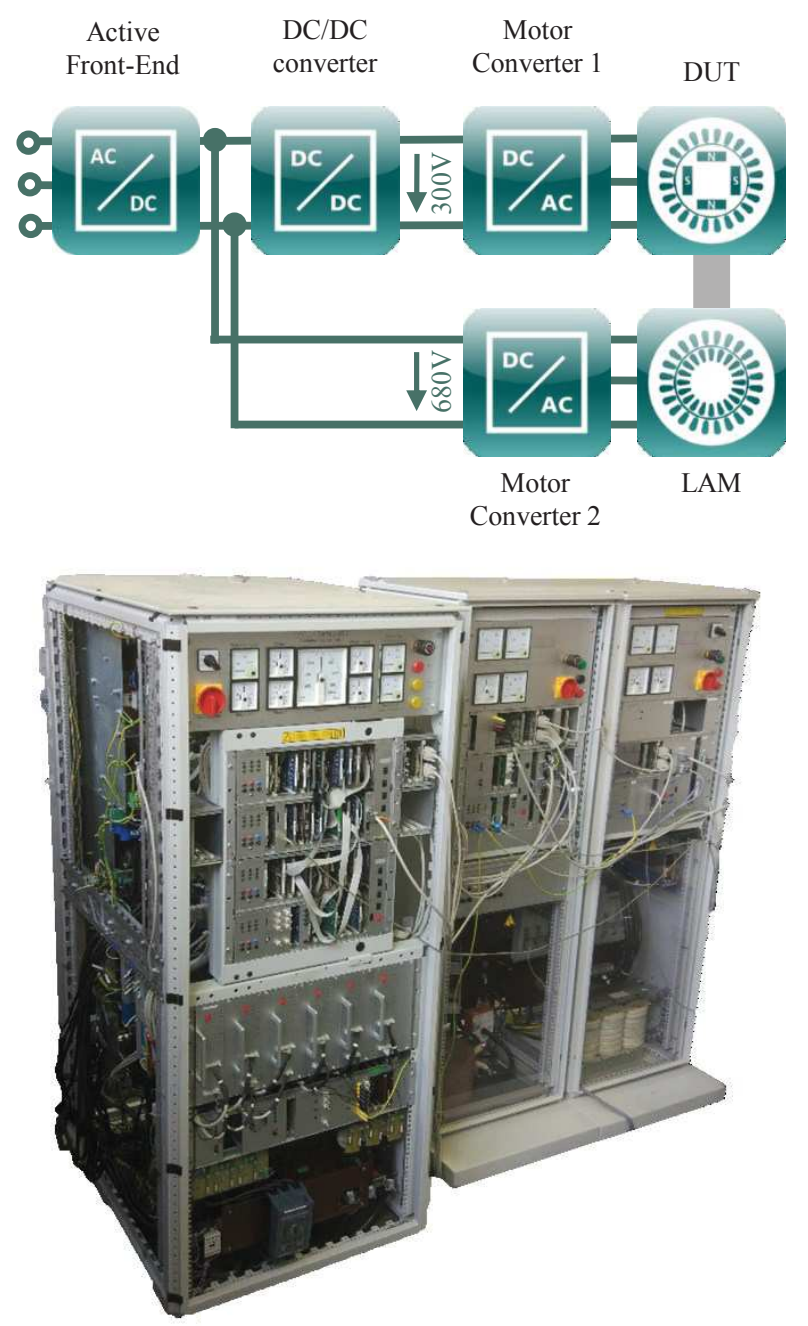

Fig. 5. Simplified schematic and photo of the test bench structure. The left cabinet shows the described MMPMC. In the middle cabinet the DC/DC converter and Motor-converter 1 for two-level operation, in the right cabinet the active front-end and Motor-converter 2 are housed.

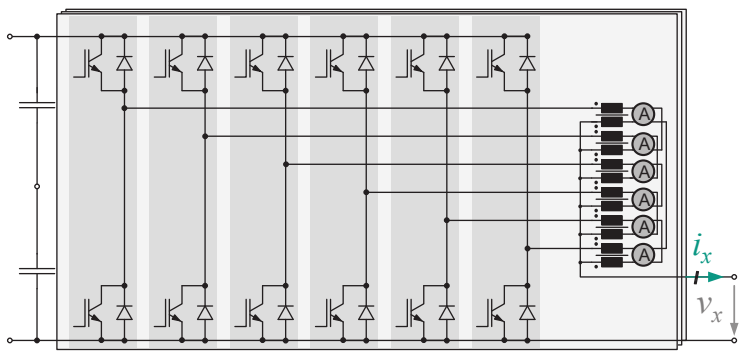

Fig. 6. Schematic diagram of the seven-level Modular Multiphase Multilevel Converter

\section{EXPERIMENTAL RESULTS}

\section{A. Current Waveform}

In Fig. 7 measurements of two- and seven-level operation at nominal torque and $2000 \mathrm{rpm}$ are given. A scope of one fundamental period of the machine's currents is shown in the upper half, a zoomed-in view is shown in the lower half. The zoomed area is indicated by a black rectangle. As it can be seen, there is a significant reduction 
in the harmonic content of the current. A considerable current ripple of $23 \mathrm{~A}$ in two-level operation, superimposed by $4 \mathrm{~A}$ noise, is reduced to a no longer identifiable current ripple.

\section{B. Comparison between Simulation and Measurement}

Generally, the behaviour of the THD shows a good accordance between simulation and measurement: Fig. 8 shows, that the highest THD is observed at small currentamplitudes and medium speed ranges. At lower speeds, only small duty-cycles are generated, therefore the currentrise is performed in short time segments. The duty cycle increases with increasing speed of the DUT, which results in an appropriate rise of the current ripple. With a rising current amplitude (higher torque demand as well as negative d-current when exceeding nominal speed range), the THD decreases due to the fact that the current amplitude increases faster than the current ripple. Furthermore, the THD is reduced drastically in seven-level operation and stays below $1.2 \%$ in every measured operating point.

Nevertheless, in two-level operation, the measured absolute THD values are visibly greater than the simulated ones as the flux-linkages in simulation are fundamental ones. Harmonics because of slotting, eccentricities or asymmetries are not considered in the simulation model.

\section{Additional Copper Losses}

Inverter-caused copper losses of the investigated IPMSM are very low, because the current ripple is low even in two-level operation. For example, at 2000rpm and $110 \mathrm{Nm}$, the THD-value of the current is $2.5 \%$, the maximum current ripple is $14 \mathrm{~A}$ and the fundamental current amplitude is $218 \mathrm{~A}$. Therefore, $\frac{1}{T_{\text {per }}} \int_{0}^{T_{\text {per }}} i^{2}(t) \mathrm{d} t$ is 1.00063 times $\frac{1}{T_{\text {per }}} \int_{0}^{T_{\text {per }}} i_{\text {fundamental }}^{2}(t) \mathrm{d} t$, thus the additional copper losses in this specific operating point amounts to $0.063 \%$ of the fundamental copper losses. Due to the quadratic interrelation of the current in copper losses, harmonic loss shares are low. In measurement, additional copper losses were less than $1 \%$ of the overall losses in every measured operating point.

\section{Additional Magnetic Losses}

The observed additional magnetic losses are given in Fig. 9 for the whole operating area. The values are consistent with the characteristic of the THD, e.g. the middle speed sector shows the highest loss share, due to a high THD-value. Measured operating points are marked with a black dot, operating points not included due to measurement uncertainties are marked with a red cross. The surface is generated using a local regression algorithm in Matlab.

Additional magnetic losses vary between $0 \%$ and $10 \%$ of the total machine losses. In most operating points, the additional magnetic losses vary between $2 \%$ and $4 \%$, the $10 \%$ value at $2000 \mathrm{rpm}$ and $27.5 \mathrm{Nm}$ is identified as outlier. Especially with lower torque values, the additional magnetic losses have to be considered as the fundamental copper and iron losses are small due to the low fundamen-
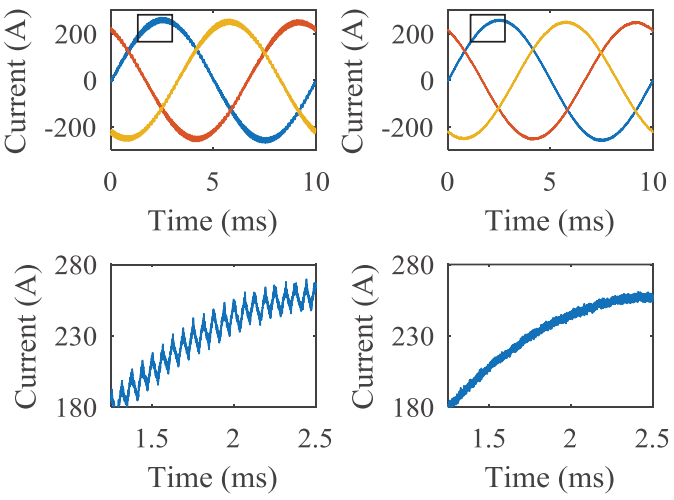

Fig. 7. Current waveforms

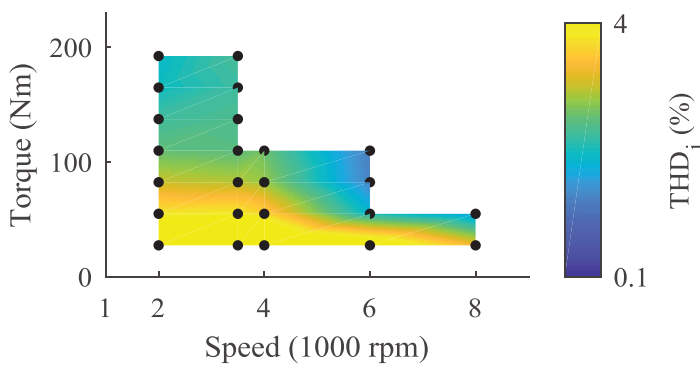

(a) Two-level operation

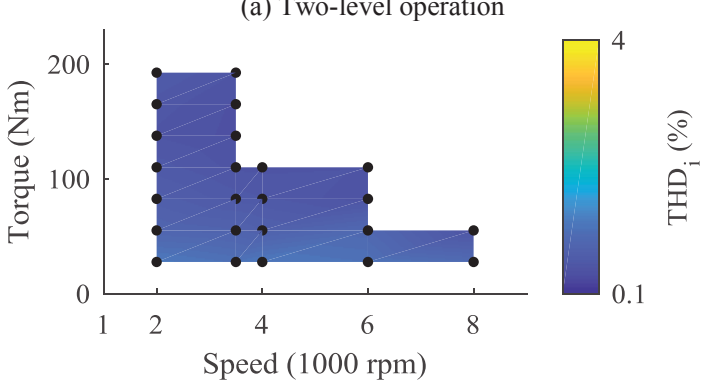

(b) Seven-level operation

Fig. 8. Machine currents THD, markers represent measured operating points

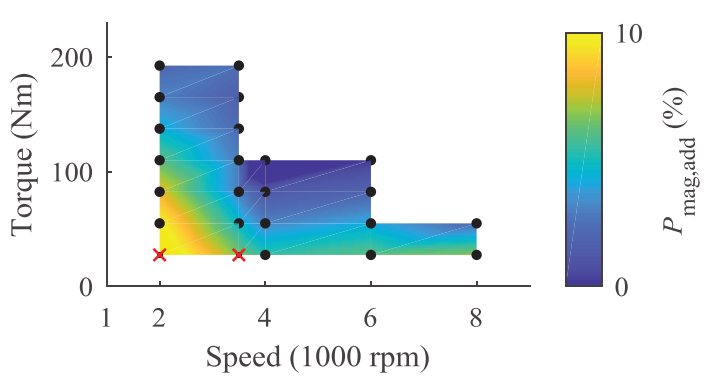

Fig. 9. Additional magnetic losses due to two-level supply

tal current values. With a higher torque demand, fundamental losses increase faster than additional losses in the magnetic domain, resulting in a decrease of the total loss share. Overall, the possible improvement in machine efficiency is lower than $1 \%$. 


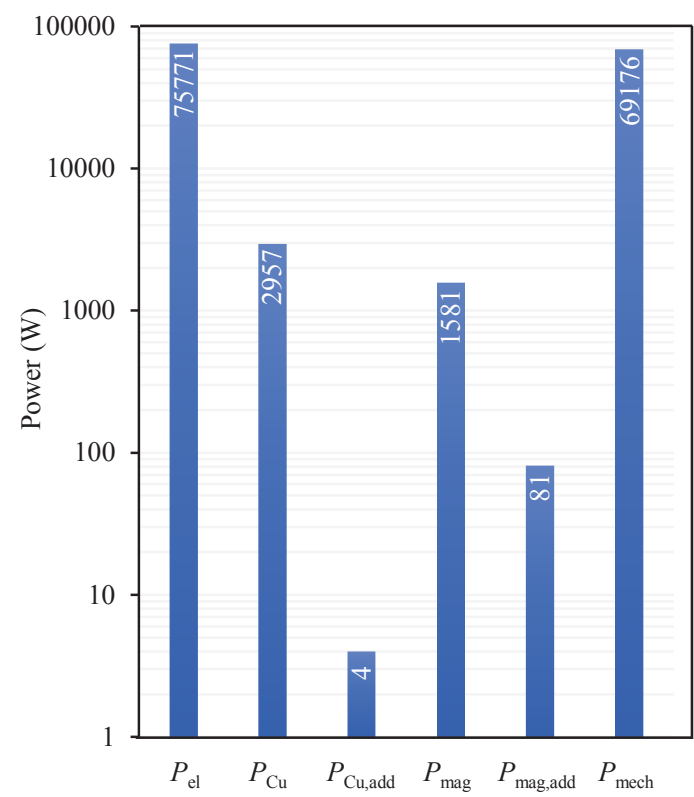

Fig. 10. Power distribution for $3500 \mathrm{rpm}$ and $192.5 \mathrm{Nm}$.

\section{E. Determination of Loss Distribution}

The determination of the loss shares is based on the method presented in [18]. According to this publication, loss shares in specific operating points are determined by considering two magnetically complementary operating points. By switching from motor to generator operation, the internal torque and the fundamental magnetic losses remain the same, on the condition that the flux values remain the same. Operation with the same flux is achieved by use of a superimposed voltage control. As a result, fundamental copper and magnetic losses are identifiable in every operating point. The method is used to identify fundamental loss shares of the machine.

Inverter-caused copper and magnetic losses are determined by comparing two- and seven-level operation. Copper losses are calculated after (3). Due to the low THD-values, additional copper losses can be neglected, see III. To identify the additional magnetic losses, the losses in two and seven-level operation is compared:

$$
\begin{aligned}
& P_{2 \mathrm{~L}, \mathrm{add}}=P_{\mathrm{loss}, 2 \mathrm{~L}}-P_{\text {loss }, 7 \mathrm{~L}} \\
& \quad=\left(P_{\mathrm{in}, 2 \mathrm{~L}}-P_{\text {mech }, 2 \mathrm{~L}}\right)-\left(P_{\mathrm{in}, 7 \mathrm{~L}}-P_{\text {mech,7L }}\right)
\end{aligned}
$$

$\begin{array}{crrr}\text { whereby input power } & P_{\text {in }} & \text { equals } \\ \sum_{x \in\{\mathrm{U}, \mathrm{V}, \mathrm{W}\}} \frac{1}{T_{\text {per }}} \int_{0}^{T_{\text {per }}} v_{x}(t) \cdot i_{x}(t) \mathrm{d} t & \text { and } P_{\text {mech }} & \text { equals }\end{array}$ the mechanical output power $T \cdot \Omega . \Omega$ is the mechanical angular frequency, $T$ the torque of the machine. Additional mechanical losses from two- or seven-level operation are not observed, as the inertia of the machine effectively damps these high frequency components. As additional copper losses can be neglected, $P_{2 \mathrm{~L}, \text { add }}$ equals the additional magnetic losses in two-level operation:

$$
P_{\text {mag,add }}=P_{2 \mathrm{~L}, \text { add }}-\underbrace{P_{2 \mathrm{~L}, \mathrm{Cu}, \text { add }}}_{\sim 0}=P_{2 \mathrm{~L}, \text { add }}
$$

Fig. 10 shows the power distribution at $n=$ $3500 \mathrm{rpm}$ and $T=192.5 \mathrm{Nm}$. The power is given in logarithmic scale for sake of a clearer presentation. The required input power $P_{\mathrm{el}}$ equals $77.8 \mathrm{~kW}$ whereas the output power $P_{\text {mech }}$ is $69.2 \mathrm{~kW}$. In this specific operating point, fundamental copper losses make up $45 \%$ and fundamental magnetic losses $25 \%$ of the overall losses. Additional magnetic losses $P_{\text {mag,add }}$ are $81 \mathrm{~W}-$ approximately $5 \%$ of the total magnetic losses $P_{\text {mag }}$ and $\sim 1 \%$ of the total losses $P_{\text {loss }, 2 \mathrm{~L}}$.

\section{CONCLUSION}

The comparison of simulations and experiments showed deviations in THD due to slotting effects of the machine. Thus, additional harmonics in current were present in experiments. Control approaches like repetitive control algorithms can be applied to the testbench to improve the current waveforms and mitigate the lower frequent harmonic parts in the current [19]. As the harmonic effects are the same for both voltage supplies, they are negligible for the considerations in this paper and with regard to the additional losses.

Simulations and measurements show a significant reduction of the machine's current ripple when using a multilevel-voltage source. Consequently the investigated seven-level MLC is a suitable converter to measure fundamental parameters of electric machines, which is proven by measurements in this work. In comparison to sinusoidal sources like speed controlled synchronous machines, current control even in saturation or a mitigation of current harmonics is possible with the MLC.

Losses in the magnetic domain of the machine reduce due to multilevel excitation. In the given operating point, a reduction of $1 \%$ is observed. Regarding operation points with smaller torque loads, the loss share of the additional magnetic losses can increase. Copper losses are less affected. Only a reduction of less than $1 \%$ compared to the total machine losses is observed. Due to its high costs, volume and weight using an MLC as a traction drive converter is not reasonable. However, in research and development of electrical machines, dynamic voltage sources with low distortions are necessary, e.g. enabling ideal sinusoidal machine currents to determine fundamental parameters and losses. As a result, direct comparisons to simulations like finite element calculation become possible.

The possibility to distinguish between fundamental and inverter-caused magnetic losses gives the opportunity to improve simulations - finite element models as well as precise lumped parameter models. The separation between iron and magnet loss shares can be realized with an appropriate finite-element model and is part of future research.

\section{REFERENCES}

[1] J. Kolb, F. Kammerer, M. Gommeringer, and M. Braun, "Cascaded Control System of the Modular Multilevel Converter for Feeding Variable-Speed Drives," IEEE Trans. Power Electron., vol. 30, no. 1, pp. 349-357, 2015. 
[2] A. Antonopoulos et al., "Modular Multilevel Converter AC Motor Drives With Constant Torque From Zero to Nominal Speed," IEEE Trans. on Ind. Applicat., vol. 50, no. 3, pp. 1982-1993, 2014.

[3] M. Hiller, D. Krug, R. Sommer, and S. Rohner, "A new highly modular medium voltage converter topology for industrial drive applications," in Proceedings of European Conference on Power Electronics and Applications, 2009.

[4] J.-J. Jung, H.-J. Lee, and S.-K. Sul, "Control Strategy for Improved Dynamic Performance of Variable-Speed Drives With Modular Multilevel Converter," IEEE J. Emerg. Sel. Topics Power Electron., vol. 3, no. 2, pp. 371-380, 2015.

[5] A. Antonopoulos, G. Mörée, J. Soulard, L. Ängquist, and H.-P. Nee, "Experimental Evaluation of the Impact of Harmonics on Induction Motors Fed by Modular Multilevel Converters: 2 - 5 Sept. 2014, Berlin, Germany ; proceedings," in Proceedings of International Conference on Electrical Machines (ICEM), Berlin, Germany, 2014, pp. 768-775.

[6] C. Bassi, M. Filippo, D. Giulivo, and A. Tessarolo, "Experimental Assessment of Medium-Voltage Induction Motor Performance Under Multilevel PWM Inverter Supply," in Proceedings of International Symposium on Power Electronics, Electrical Drives, Automation and Motion (SPEEDAM), 2012, pp. 253-258.

[7] P. Rasilo et al., "Effect of Multilevel Inverter Supply on Core Losses in Magnetic Materials and Electrical Machines," IEEE Trans. Energy Convers., vol. 30, no. 2, pp. 736-744, 2015.

[8] M. Schweizer and J. W. Kolar, "High efficiency drive system with 3-level T-type inverter," in Proceedings of European Conference on Power Electronics and Applications, 2011.

[9] W. Tong, Y. Wang, R. Sun, S. Wu, and J. Jia, "Simulation and Experimental Study on No-Load Loss Distributions of an IPM Motor Under the Conditions of Both Sinusoidal Supply and Converter Supply," IEEE Trans. Magn., vol. 54, no. 11, pp. 1-6, 2018.

[10] G. Bertotti, Hysteresis in magnetism: For physicists, materials scientists and engineers. San Diego, Calif.: Acad. Press, 1998.
[11] F. Preisach, "Über die magnetische Nachwirkung," 1935.

[12] C. P. Steinmetz, "On the law of hysteresis," Proc. IEEE, vol. 72, no. 2, pp. 197-221, 1984.

[13] L. R. Dupre and J. Melkebeek, "Electromagnetic hysteresis modelling: from material science to finite element analysis of devices," International Compumag Society Newsletter, vol. 2003, pp. 4-15, 2003.

[14] J. Richter, P. Winzer, and M. Doppelbauer, "Einsatz virtueller Prototypen bei der akausalen Modellierung und Simulation von permanenterregten Synchronmaschinen," in Beiträge des Internationalen ETG-Kongresses vom 5. - 6. November 2013 in Berlin:

Energieversorgung auf dem Weg nach 2050.

[15] A. Schmitt, M. Gommeringer, J. Kolb, and M. Braun, "A High Current, High Frequency Modular Multiphase Multilevel Converter for Power Hardware-in-the-Loop Emulation," in Proceedings of PCIM Europe, 2014.

[16] C. Axtmann, M. Boxriker, and M. Braun, "A custom, high-performance real time measurement and control system for arbitrary power electronic systems in academic research and education," in 2016 18th European Conference on Power Electronics and Applications (EPE'16 ECCE Europe): 5-9 Sept. 2016, Karlsruhe, 2016, pp. 1-7.

[17] J. Richter, T. Gemassmer, and M. Doppelbauer, "Predictive current control of saturated crosscoupled permanent magnet synchronous machines," in Proceedings of International Symposium on Power Electronics, Electrical Drives, Automation and Motion (SPEEDAM), Ischia, Italy, 2014, pp. 830-835.

[18] J. Richter, A. Dollinger, and M. Doppelbauer, "Iron loss and parameter measurement of permanent magnet synchronous machines," in Proceedings of International Conference on Electrical Machines (ICEM), Berlin, Germany, 2014, pp. 1635-1641.

[19] J. Richter, T. Lannert, T. Gemassmer, and M. Doppelbauer, "Mitigation of Current Harmonics in Inverter-Fed Permanent Magnet Synchronous Machines with Nonlinear Magnetics," in Proceedings of PCIM Europe, 2015. 